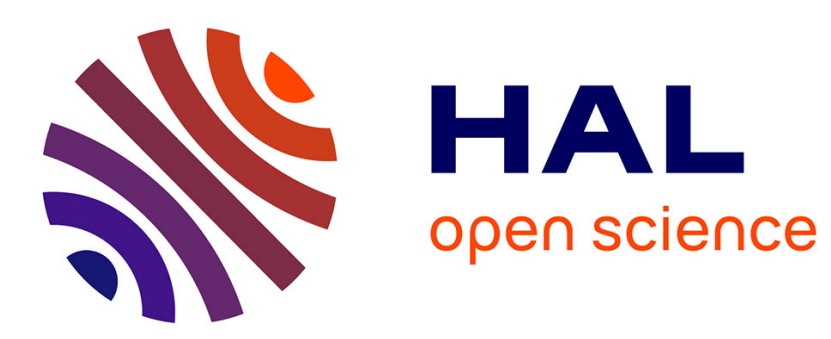

\title{
Improving nuclear mass predictions through the Garvey-Kelson relations
}

\author{
I.O. Morales, A. Frank
}

\section{To cite this version:}

I.O. Morales, A. Frank. Improving nuclear mass predictions through the Garvey-Kelson relations. Physical Review C, 2011, 83, pp.054309. 10.1103/PhysRevC.83.054309 . in2p3-00601000

\section{HAL Id: in2p3-00601000 https://hal.in2p3.fr/in2p3-00601000}

Submitted on 20 Jun 2011

HAL is a multi-disciplinary open access archive for the deposit and dissemination of scientific research documents, whether they are published or not. The documents may come from teaching and research institutions in France or abroad, or from public or private research centers.
L'archive ouverte pluridisciplinaire HAL, est destinée au dépôt et à la diffusion de documents scientifiques de niveau recherche, publiés ou non, émanant des établissements d'enseignement et de recherche français ou étrangers, des laboratoires publics ou privés. 


\title{
Improving nuclear mass predictions through the Garvey-Kelson relations.
}

\author{
Irving O. Morales ${ }^{1}$ and A. Frank ${ }^{2}$ \\ ${ }^{1}$ Grand Accélérateur National d'Ions Lourds (GANIL), \\ CEA/DSM-CNRS/IN2P3, Bvd Henri Becquerel, 14076 Caen, France \\ ${ }^{2}$ Instituto de Ciencias Nucleares, Universidad Nacional Autónoma de México, \\ Apartado Postal 70-543, 04510 México, D.F., Mexico
}

(Dated: April 27, 2011)

\begin{abstract}
We describe a procedure to improve the nuclear mass predictions obtained by means of the Garvey-Kelson (GK) iterative process. This is achieved by reducing the degrees of freedom involved in the process, expressing the Garvey-Kelson relations in terms of one-neutron and one-proton separation energies and using a theoretical estimation for them. This approach has the effect that the intrinsic error associated to the iterative process grows significantly more slowly. This leads to a better quality of the nuclear mass predictions and therefore extends the range for which the predictions are accurate. We test these ideas using different mass models.
\end{abstract}

PACS numbers: 21.10.Dr,21.60.-n

\section{INTRODUCTION}

A precise knowledge of nuclear masses in regions away from measured values is an important topic in nuclear physics, due to their importance in astrophysical calculations [1], but a large amount of these masses remain unknown. The values of the unknown nuclear masses are obtained through theoretical predictions but, unfortunately, there is a lack of consensus and most predictions differ drastically from each other, specially in the region of large neutron excess $[1,2]$. There are a large diversity of models and techniques to predict nuclear masses [1] which can essentially be classified into global and local approximations.

Global models use the totality of currently available experimental information corresponding to the measured nuclear masses. Examples of this kind of models are the liquid drop model [3], the finite-range droplet model [4], the Duflo-Zuker model [5], the Hartree-Fock approach [6] and the CLEAN deconvolution algorithm [7]. These models assume that the current experimental knowledge is sufficient to determine the necessary parameters of the theory, so they can be used to describe the behavior of the masses outside the known region. The models thus focus on explaining the general characteristics and overall systematics of the mass surface on the $\mathrm{N}, \mathrm{Z}$ plane. While it is true that it takes a great effort to capture the main properties of these global systematics, in order to predict such a large amount of information, small details and surface structures at smaller scales are usually not possible to describe. These "higher resolution" structures, however, are required to provide sufficient accuracy in the predictions, needed for most practical applications [1].

On the other hand, local approaches view the problem with a completely different philosophy. These techniques attempt to predict nuclear masses much more precisely, but in a limited region close to the experimentally known one. These models predict one nucleus at a time and consider only the information arising from the neighboring nuclei, i.e. the structures in the immediate vicinity and how they vary as a function of the number of neutrons and protons. Essentially there are two methods to predict nuclear masses in this fashion. The Audi-Wapstra extrapolation method [8], based on the systematics and smoothness of the mass surface and its derivatives, the one and two particle separation energies and the $Q_{\beta}$ and $Q_{\alpha}$ decay values. The second one arises from the use of the Garvey-Kelson relations [9], and in this article we will concentrate on them. The GK relations are algebraic equations between the masses of six neighboring nuclei obtained by considering an extreme single-particle model, where the interactions between neutrons and protons vary smoothly along the $(\mathrm{N}, \mathrm{Z})$ plane. These relations are obtained by constructing combinations of neighboring masses in such a way that the residual interactions cancel out. The expressions obtained in this way are the following

$$
\begin{gathered}
M(N+2, Z-2)-M(N, Z) \\
+M(N, Z-1)-M(N+1, Z-2) \\
+M(N+1, Z)-M(N+2, Z-1)=0 \\
M(N+2, Z)-M(N, Z-2) \\
+M(N+1, Z-2)-M(N+2, Z-1) \\
+M(N, Z-1)-M(N+1, Z)=0,
\end{gathered}
$$

where $M(N, Z)$ is the nuclear mass with $\mathrm{N}$ neutrons and $\mathrm{Z}$ protons. Two restrictions must be taken into account : none of the nuclei in the equations should have $N=Z$ odd and, for relation 1 to be satisfied is necessary that $N>Z$, to secure a proper cancelation of the isospin dependence of the residual interactions [9]. 
Using either of these relationships it is possible to predict the value of a given nuclear mass if the other five are known. Varying the position of the mass to be predicted and using both GK relations, it is possible to obtain several predictions for the same mass, as long as the neighboring nuclear masses are known. This technique has been used previously in an iterative fashion to predict whole regions in the N,Z plane [10], using the predicted nuclear masses as known values for the next iterations. The main drawback with this procedure is that the GK relations are not satisfied exactly by the nuclear masses. The average error in the GK relations for the experimentally known nuclear masses is of about $100 \mathrm{keV}$. In fact, we have found no evidence of the error growing when we go further away from the region of stability [11]. The problem arises when the relations are used to predict in an iterative fashion since, as the number of iterations increases, the intrinsic error grows rapidly. After a few iterations the deviation on the predictions is larger than the desired precision. An extensive analysis of how the error on the prediction grows as a function of the iterations has been done in [10]. In this paper we propose a way to improve the predictions of the Garvey-Kelson relations, something we achieve by first modifying the iterative process to predict more nuclear masses per iteration, as we explain below.

\section{THE GARVEY-KELSON RELATIONS AND THE SEPARATION ENERGIES}

The Garvey-Kelson relations involve five degrees of freedom, which are not independent of each other. Through the iterative process each of these degrees of freedom accumulates error, leading to a growing innacuracy. It is possible, however, to reduce the error in the process by modifying the way in which the different degrees of freedom are related to each other. The first Garvey-Kelson relation, eq.1 can be expressed in two ways, in terms of the separation energies of neutrons and protons

$$
\begin{aligned}
S_{n}(N+2, Z-2)+S_{n}(N+1, Z) & \\
+M(N, Z-1)-M(N+2, Z-1) & =0, \\
S_{p}(N+2, Z-1)+S_{p}(N, Z) & \\
-M(N+1, Z)+M(N+1, Z-2) & =0,
\end{aligned}
$$

where

$$
\begin{aligned}
& S_{n}(N, Z)=M(N, Z)-M(N-1, Z), \\
& S_{p}(N, Z)=M(N, Z)-M(N, Z-1) .
\end{aligned}
$$

In the same fashion, it is possible to express the second Garvey-Kelson relation as

$$
\begin{aligned}
S_{n}(N+2, Z)+S_{n}(N+1, Z-2) & \\
-M(N+2, Z-1)+M(N, Z-1) & =0, \\
S_{p}(N+2, Z)+S_{p}(N, Z-1) & \\
+M(N+1, Z-2)-M(N+1, Z) & =0 .
\end{aligned}
$$

The former expressions relate one-nucleon separation energies with mass values. If we can obtain the values of the separation energies, then it is possible to reduce the number of degrees of freedom at play. Assuming that we know the value of the separation energies and using equations $3,4,7$ and 8 , there are 8 possible ways of estimating the value of a given nuclear mass (predicted mass) in terms only of the value of another mass (generator mass). The separation energies function as a backbone for the prediction, so we require these energies to use this technique. One may think that determining the separation energies is equivalent to knowing the nuclear masses, but this is far from being correct. The separation energies can be considered as the discrete derivatives of the mass surface, so in order to obtain the value of the actual surface it is necessary to integrate them and the corresponding integration constants are needed. Although it is possible to use the known experimental masses as integration constants, the error due to this process grows very rapidly.

Once the separation energies have been determined, the iteration procedure works in the same way as in the one described in [10]. In each iteration there are nuclei that meet 2 conditions, 1) the value of the nuclear mass to be determined is unknown and 2) we do know the value of a neighboring nuclear mass, involved in at least one of the relations expressed on eqs. 3, 4, 7 and 8. On every nucleus that meets these conditions, we calculate as many estimates as possible, averaging these values in order to obtain a prediction for the unknown mass of the nucleus. The mass values predicted on previous iterations are used as known values for the next iteration. It is important to note that the values used for the separation energies are defined from the beginning and do not change throughout the procedure, so the number of degrees of freedom which can accumulate error is reduced to one per relation. Having reliable predictions for the separation energies is thus crucial.

The new iterative procedure (IGK2), which uses equations 3, 4, 7 and 8, has several advantages over the old iterative process (IGK1), defined in terms of the original Garvey-Kelson relations. The main advantage is the smaller number of iterations necessary to reach a given nucleus. While IGK1 requires previous knowledge of at least 5 neighboring masses in order to predict a new one, we only need one mass in IGK2 (plus the 
information provided by the one-nucleon separation energies, of course). Since the condition of knowing one mass is satisfied more often than knowing 5 masses, the number of nuclei predicted is greater in the new process. It has been shown previously that the error on the predictions grows systematically on each iteration [10], which implies that if a given nucleus is reached in less iterations, the mass prediction of that nucleus will have a smaller accumulated error. This effect can be clearly seen in table I, which compares the number of nuclei for which the mass is predicted per iteration for both processes. In both cases the iterative process starts from the masses reported on the AME03 compilation [8]. A graphical comparison of the two processes and the regions covered after the first 10 iterations is shown in figure 1. It is clear from the table and the figure that the IGK2 iterative process is significantly more effective.

TABLE I: Number of nuclei predicted per iteration on the first 10 iterations of the process using the original

Garvey-Kelson relations (IGK1) and the iterative process using the modified Garvey-Kelson relations of equations 3, 4, 7 and 8 (IGK2).

\begin{tabular}{ccccccccccc}
\hline \hline Iteration & 1 & 2 & 3 & 4 & 5 & 6 & 7 & 8 & 9 & 10 \\
\hline IGK1 & 163 & 131 & 135 & 134 & 133 & 131 & 132 & 132 & 131 & 126 \\
\hline IGK2 & 560 & 516 & 494 & 486 & 465 & 428 & 375 & 338 & 306 & 277 \\
\hline \hline
\end{tabular}

An additional advantage provided by this technique arises from the relative position of the predicted and the generator mass. These two masses always belong to nuclei in the same set of isotopes or isotones, i.e., each of the equations relate two masses with the same number of neutrons or protons. This allows the predictions to advance directly towards the proton- or neutron-rich regions. The IGK1 procedure, in contrast, moves forward on the $\mathrm{A}=\mathrm{N}+\mathrm{Z}$ or $\mathrm{T}=(\mathrm{N}-\mathrm{Z}) / 2$ directions, making it less efficient. Finally, the nature of the error implies an additional advantage for IGK2, since part of the deviation comes form the separation energy estimation, which can be improved systematically with better analyses of these energies, using both global and local approaches.

\section{PREDICTION TEST}

The IGK1 iterative procedure has been tested in [10]. In order to quantitatively compare it to the new iterative process, we use the same set of data. The test consists in dividing the experimentally known masses into two subsets, one used to fit the model and the other to be compared to the predictions. Specifically, the reported masses on the AME03 compilation [8] have been divided into a subset conformed by the masses measured up to
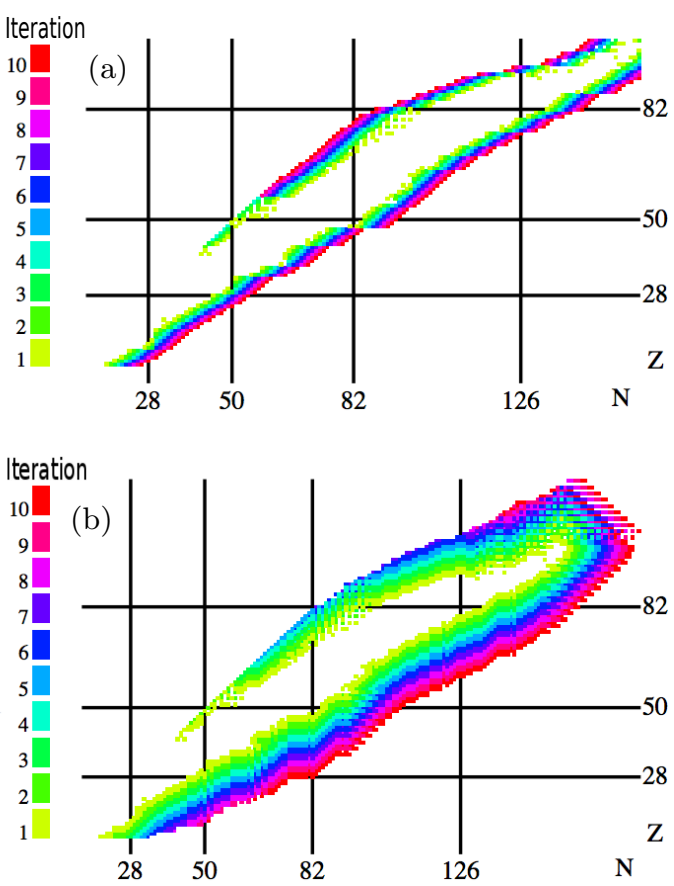

FIG. 1: (Color online) Nuclei predicted per iteration in the first 10 iterations of the process using (a) the original Garvey-Kelson relations (IGK1) and (b) the modified Garvey-Kelson relations defined on equations $3,4,7$ and 8 (IGK2).

the year 1995, i.e. reported in the AME95 compilation [12], and a prediction subset comprised by those masses present on the AME03 compilation but absent on AME95. For this test we only consider nuclei with $N \geq 28$ and $Z \geq 28$.

The first step to apply the IGK2 process is to estimate the experimental separation energies. As should be evident, the quality of the mass predictions depends on an accurate description of them. We stress that the main reason for which a better accuracy is possible to achieve with this procedure, is that the $(\mathrm{N}, \mathrm{Z})$ surface produced by the separation energies has simpler systematics than the mass surface. In addition, the one-nucleon separation energies can be directly measured experimentally and do not require, as the masses, a subsequent analysis which may introduce further systematic errors. As a first approximation we take the simple parametrization described in [13] which assumes that the one-neutron separation energies can be parametrized as

$$
\begin{aligned}
S_{n}(N, Z)= & \left(a n_{1}+a n_{2} A^{1 / 3}\right)\left(\frac{N}{Z}\right)^{-1} \\
& -a n_{3}+a n_{p} \frac{\delta(N)}{A^{1 / 2}}-a n_{s} \mu(N),
\end{aligned}
$$

where $A=N+Z$, the $a n_{p}$ term models the effects 
of pairing, $\delta(N)=1$ if the neutron number is even or $\delta(N)=-1$ if it is odd. The $a n_{s}$ term is a correction for the nuclear shell effects which subtracts a constant energy on every shell, the value of $\mu$ depends on the shell, $\mu(N)=0$ if $1 \leq N \leq 28, \mu(N)=1$ if $29 \leq N \leq 50$, $\mu(N)=2$ if $51 \leq N \leq 82, \mu(N)=3$ if $83 \leq N \leq 126$ and $\mu(N)=4$ if $127 \leq N$.

The one-proton separation energy parametrization is the same, but changing $N$ by $Z$ and adding an expression to model the energy due to the Coulomb interaction. Including this term the parametrization can be expressed as

$$
\begin{aligned}
S_{p}(N, Z)= & \left(a p_{1}+a p_{2} A^{1 / 3}\right)\left(\frac{Z}{N}\right)^{-1} \\
& -a p_{3}+a p_{p} \frac{\delta(Z)}{A^{1 / 2}} \\
& -a p_{s} \mu(Z)-a p_{c} \frac{Z}{A^{1 / 3}},
\end{aligned}
$$

where $\delta(Z)$ and $\mu(Z)$ has the same behavior as $\delta(N)$ and $\mu(N)$.

The validity of these equations to model separation energies has been quantified in [13]. In order to use these estimations on the iterative process the values of the parameters involved on equations 9 and 10 have been obtained fitting the models to the known separation energies, obtained through the experimentally known nuclear masses on the fitting subset. The values obtained for the parameters are shown on table II. Once the separation energies have been determined, the iterative procedure can be applied.

TABLE II: Values of the parameters obtained for the one-neutron separation energies, eq. 9 and for the one-proton separation models, eq. 10

\begin{tabular}{cccccc}
\hline \hline$a n_{1}$ & $a n_{2}$ & $a n_{3}$ & $a n_{p}$ & $a n_{s}$ & \\
5.178 & 3.549 & 5.354 & 11.169 & 1.566 & \\
\hline$a p_{1}$ & $a p_{2}$ & $a p_{3}$ & $a p_{p}$ & $a p_{s}$ & $a p_{c}$ \\
11.120 & 1.054 & 1.043 & 12.473 & 1.474 & 1.111 \\
\hline \hline
\end{tabular}

On each iteration two quantities are calculated, the root mean square deviation for the nuclei predicted on that iteration $\sigma$ and the root mean square deviation for the nuclei predicted up to that iteration $A \sigma$. These quantities, for the $i^{\prime}$ th iteration, are defined as follows

$$
\sigma^{i}=\left(\frac{1}{N_{i}} \sum_{j=1}^{N_{i}}\left(M_{i, j}^{G K}-M_{i, j}^{\exp }\right)^{2}\right)^{1 / 2}
$$

$$
A \sigma^{i}=\left(\frac{1}{\sum N_{i}} \sum_{i=1}^{n_{i}} \sum_{j=1}^{N_{i}}\left(M_{i, j}^{G K}-M_{i, j}^{e x p}\right)^{2}\right)^{1 / 2}
$$

where the index $j$ goes over the $N_{i}$ nuclei predicted on iteration $i$. The nuclear mass value obtained by this method is $M^{G K}$, while the experimental value for that nuclear mass is $M^{e x p}$.

Table III shows the performance of IGK2 for the nuclei predicted at each iteration in the prediction subset. The same table compares this prediction with the one obtained with IGK1 on the same nuclei. The number of nuclei predicted on the prediction subset is shown for each iteration as well as the number of nuclei predicted up to that iteration, shown in parenthesis. It is important to notice that after only two iterations the IGK2 process has covered almost all nuclei on the prediction subset, 319 of 371 nuclei. On each iteration both the root mean square deviation per iteration $\sigma$ and the accumulated deviation $A \sigma$ are lower in IGK2. In the case of the first iteration which includes 227 nuclei, the root mean square deviation is lower by $50 \%$.

TABLE III: Root mean square deviations $\sigma^{i}$ and $A \sigma^{i}$ in $\mathrm{MeVs}$ for the nuclei predicted on each iteration of IGK2 and the predictions obtained with the IGK1 process. The second row shows the number of nuclei predicted on iteration $i$ and in parenthesis the number of nuclei predicted up to iteration $i$. Both numbers are constrained to belong to the prediction subset.

\begin{tabular}{rccccccccc}
\hline \hline \multicolumn{1}{c}{ Iteration 1 } & Iteration 2 & Iteration 3 & Iteration 4 \\
\hline 227 & $(227)$ & 92 & $(319)$ & 34 & $(353)$ & 10 & $(363)$ \\
$\sigma^{i}$ & $A \sigma^{i}$ & $\sigma^{i}$ & $A \sigma^{i}$ & $\sigma^{i}$ & $A \sigma^{i}$ & $\sigma^{i}$ & $A \sigma^{i}$ \\
\hline IGK2 & 0.445 & 0.445 & 0.824 & 0.580 & 1.129 & 0.653 & 1.300 & 0.679 \\
\hline IGK1 & 1.002 & 1.002 & 0.925 & 0.981 & 1.536 & 1.047 & 2.517 & 1.108 \\
\hline \hline
\end{tabular}

These results show that through the use of separation energy information in the Garvey-Kelson relations, it is possible to significantly improve the predictive power of the iterative process, thus improving the range for which the predictions are accurate. However, the model we have considered for the separation energies, eq. 9 and 10 , is very simple. We now show that the iterative process can be further improved by introducing better estimations of the separation energies. As an example, we will use the separation energies obtained in terms of the predictions of the Duflo-Zuker mass model. We call this iterative process IGKDZ. In order to test the accuracy of this procedure we have again subjected it to the same test as before. Table IV displays the 
results. The table shows the deviation $\sigma$ per iteration as well as the accumulated deviation $A \sigma$ for the IGKDZ process. The same quantities have been computed for the predictions obtained directly with the Duflo-Zuker mass model on those nuclei, in order to compare both methodologies.

TABLE IV: Root mean square deviations $\sigma^{i}$ and $A \sigma^{i}$ in $\mathrm{MeVs}$ for the nuclei predicted on each iteration of the iterative process IGKDZ and the predictions obtained directly with the Duflo-Zuker model. The second row shows the number of nuclei predicted on iteration $i$ and in parenthesis the number of nuclei predicted up to iteration $i$. Both numbers are constrained to nuclei within the subset of prediction masses.

\begin{tabular}{ccccccccc}
\hline \hline \multicolumn{1}{c}{ Iteration 1 } & Iteration 2 & Iteration 3 & Iteration 4 \\
\hline 227 & $(227)$ & 92 & $(319)$ & 34 & $(353)$ & 10 & $(363)$ \\
$\sigma^{i}$ & $A \sigma^{i}$ & $\sigma^{i}$ & $A \sigma^{i}$ & $\sigma^{i}$ & $A \sigma^{i}$ & $\sigma^{i}$ & $A \sigma^{i}$ \\
\hline
\end{tabular}

\begin{tabular}{lllllllll} 
IGKDZ & 0.227 & 0.227 & 0.300 & 0.250 & 0.286 & 0.254 & 0.370 & 0.258 \\
\hline
\end{tabular}

$\begin{array}{lllllllll}\text { DZ } & 0.326 & 0.326 & 0.335 & 0.329 & 0.319 & 0.328 & 0.396 & 0.330\end{array}$

Comparing the results on table III and table IV we find a very significant improvement in accuracy. Furthermore, the predictions obtained with the IGK2 methodology are much better than the ones using the original Garvey-Kelson relations. On the first four iterations we observe that the results are of high quality. According to table I these four iterations cover about
2000 nuclei where the predictions are improved in comparison with the predictions of the model used to generate the separation energies. In this fashion, the new iterative process can be thought as a procedure to improve the predictive power of existing nuclear mass models.

\section{CONCLUSION}

A new way to use the Garvey-Kelson relations has been presented. This approach involves expressing the original Garvey-Kelson relations in terms of the one-neutron and one-proton separation energies. The modified GK relations are then used on an iterative fashion, helped by (either local or global) estimations for the separation energies, which are used as a backbone constraining the process. The inclusion of separation energies reduce the number of degrees of freedom involved on the iterative process and allows for a more efficient and accurate predictions. Comparisons of the original iterative process with the one proposed in this work are presented, using different estimations for the separation energies. It has been shown that the predictions using the new approach (IGK2) are more accurate and are competitive with the best predictions available [1], at least for the 2000 nuclei closest to the experimentally known region. Finally, this approach can be combined with existing nuclear mass models to significantly improve their predictive power, as demonstrated for the Duflo-Zuker model.
[1] D. Lunney, J.M. Pearson, and C. Thibault, Rev. Mod. Phys. 75, 1021 (2003).

[2] Klaus Blaum, Physics Reports 425, 1-78, (2006).

[3] C. F. von Weizsäcker, Z. Phys. 96, 431 (1935).

[4] P. Möller, J. R. Nix, W. D. Myers, and W. J. Swiatecki, At. Data Nucl. Data Tables 59, 185 (1995).

[5] J. Duflo and A. P. Zuker, Phys. Rev. C 52, R23 (1995).

[6] S. Goriely, F. Tondeur, and J. M. Pearson, At. Data Nucl. Data Tables 77, 311 (2001).

[7] Irving O. Morales, P. Van Isacker, V. Velazquez, J. Barea, J. Mendoza-Temis, J. C. Lpez Vieyra, J. G. Hirsch, and A. Frank, Phys. Rev. C 81, 024304 (2010)

[8] G. Audi, A.H. Wapstra and C.Thibault, Nucl. Phys. A
729, 337 (2003).

[9] G.T. Garvey and I. Kelson, Phys. Rev. Lett. 16, 197 (1966); G.T. Garvey, W.J. Gerace, R.L. Jaffe, I. Talmi, and I. Kelson, Rev. Mod. Phys. 41, S1 (1969).

[10] Irving O. Morales, J. C. López Vieyra, J. G. Hirsch, and A. Frank, Nucl. Phys. A 828, pp. 113-124, (2009)

[11] J. Barea, A. Frank, J. G. Hirsch, and P. Van Isacker, Phys. Rev. Lett. 94, 102501 (2005).

[12] G. Audi and A. H. Wapstra, Nucl. Phys. A 595, 409-480 (1995).

[13] K. Vogt, T. Hartmann and A. Zilges, Physics Letters B 517, 255-260 (2001) 